\title{
Dark Matter and Gauge Coupling Unification in Non-SUSY SO(10) Grand Unified Models
}

\author{
Natsumi Nagata ${ }^{* \dagger}$ \\ William I. Fine Theoretical Physics Institute, School of Physics and Astronomy, \\ University of Minnesota, Minneapolis, Minnesota 55455, USA \\ Kavli IPMU (WPI), University of Tokyo, Kashiwa, Chiba 277-8584, Japan \\ E-mail: nagat006aumn . edu
}

\begin{abstract}
The stability of dark matter is naturally explained if there is an additional U(1) symmetry which is spontaneously broken to a discrete symmetry at a high-energy scale. Such a framework is realized in the context of the $\mathrm{SO}(10)$ grand unification. In this work, we discuss dark matter models in the non-supersymmetric SO(10) grand unified models in which the stability of dark matter is assured by this mechanism. We find that the requirement of gauge coupling unification with a sufficiently high unification scale to evade the proton decay constraints plays an important role in selecting viable candidates. Some of the dark matter models can be tested in future dark matter direct searches and proton decay experiments.
\end{abstract}

18th International Conference From the Planck Scale to the Electroweak Scale 25-29 May 2015

Ioannina, Greece

\footnotetext{
* Speaker.

${ }^{\dagger}$ Based on work in collaboration with Yann Mambrini, Keith A. Olive, Jérémie Quevillon, and Jiaming Zheng [1, 2].
} 


\section{Introduction}

A variety of cosmological observations has provided strong evidences for the existence of dark matter (DM). One of the distinct properties of dark matter is its stability; for a particle to be a good DM candidate, it should be stable or have a lifetime longer than the age of the Universe. To insure the stability, it is often assumed that there exists a discrete symmetry which prevents a DM particle from decaying into the Standard Model (SM) particles. For instance, the $R$-parity in the minimal supersymmetric (SUSY) SM allows the lightest SUSY particle to be a good DM candidate. It is quite often the case, however, that the origin of such a discrete symmetry is unclear and thus its introduction seems more or less ad hoc. Thus, it is of great interest if we can find some mechanism to generate a discrete symmetry that makes DM stable.

In fact, we can easily obtain a discrete symmetry if there is an additional U(1) gauge symmetry beyond the SM gauge symmetries which is spontaneously broken at a high-energy scale. Suppose that there is a U(1) gauge symmetry for which generic fields (including SM fields) $\varphi_{i}$ and a Higgs field $\varphi_{H}$ have charges $Q_{i}$ and $Q_{H}$, respectively. Here, we normalize these charges so that they are integers. We further assume that the charge of the Higgs field $\varphi_{H}$ satisfies $Q_{H} \equiv 0(\bmod . N)$ with $N$ being a positive integer. A vacuum expectation value (VEV) of this Higgs field $\left\langle\varphi_{H}\right\rangle$ breaks the extra U(1) symmetry. An important observation here is that both the Lagrangian of the theory and the $\operatorname{VEV}\left\langle\varphi_{H}\right\rangle$ are invariant under the following transformations:

$$
\varphi_{i} \rightarrow \exp \left(\frac{i 2 \pi Q_{i}}{N}\right) \varphi_{i}, \quad\left\langle\varphi_{H}\right\rangle \rightarrow \exp \left(\frac{i 2 \pi Q_{H}}{N}\right)\left\langle\varphi_{H}\right\rangle=\left\langle\varphi_{H}\right\rangle
$$

This indicates that $\left\langle\varphi_{H}\right\rangle$ breaks the extra $\mathrm{U}(1)$ symmetry into a $\mathbb{Z}_{N}$ symmetry $[3,4,5,6,7]$.

We can consider such an extra U(1) symmetry within the context of the $\mathrm{SO}(10)$ grand unification $[8,9]$, as $\mathrm{SO}(10)$ is a rank-five gauge group. Indeed, the $\mathrm{SO}(10)$ grand unified theories (GUTs) have various attractive features. The SM quarks and leptons as well as three right-handed neutrinos are embedded into three generations of $\mathbf{1 6}$ spinor representations. Since $\mathrm{SO}(10)$ gauge theory is free from gauge anomalies, the anomaly cancellation in the SM can naturally be explained. In addition, SO(10) GUTs can realize gauge coupling unification even without SUSY with the aid of intermediate gauge symmetries (for a review of non-SUSY SO(10) GUTs with intermediate gauge symmetries, see Ref. [10]). In this case, masses of right-handed neutrinos are of the order of the intermediate gauge symmetry breaking scale $M_{\text {int }}$, which explains small neutrino masses via the seesaw mechanism $[11,12,13,14,15,16]$ if $M_{\text {int }}$ is sufficiently high.

In the non-SUSY SO(10) GUTs, the extra U(1) symmetry is broken at the intermediate scale $M_{\text {int }}$. Thus, by appropriately choosing the Higgs field that breaks the intermediate gauge symmetry, we can obtain a remnant discrete symmetry at low energies according to the mechanism discussed above. In fact, it turns out that if a VEV of a $\mathbf{1 2 6}(\mathbf{6 7 2})$ of SO(10) breaks the intermediate gauge symmetry, then a $\mathbb{Z}_{2}\left(\mathbb{Z}_{3}\right)$ symmetry appears below the intermediate scale [17]. In particular, if we restrict ourselves to consider $\operatorname{SO}(10)$ multiplets whose dimensions are $\leq 210$, then the $\mathbb{Z}_{2}$ symmetry is the only possibility to be realized in such a framework. This $\mathbb{Z}_{2}$ symmetry is found to be equivalent to the so-called matter parity, $P_{M}=(-1)^{3(B-L)}$; this is not surprising as $\mathrm{SO}(10)$ contains the $B-L$ symmetry. Since the SM fermions have the matter parity odd and the SM Higgs field has the matter parity even, a boson (fermion) with the matter parity odd (even) cannot decay 
into the SM particles in the presence of this $\mathbb{Z}_{2}$ symmetry. Thus, $\mathrm{SO}(10)$ GUTs can nicely explain the stability of DM with this remnant $\mathbb{Z}_{2}$ symmetry [18, 19, 20, 21].

In this work, we systematically study possibilities of DM in SO(10) GUT models which is stabilized with this mechanism. We find that two classes of DM candidates can be realized in this setup; one is the non-equilibrium thermal DM (NETDM) [22] and the other is the weaklyinteracting massive particles (WIMPs). The former DM is a SM singlet fermion which is nonthermally produced from the scattering of SM particles in the thermal bath through the intermediate/GUT scale particle exchange. We discuss this case in the next section following the study in Ref. [1]. For the latter case, DM can be either a scalar or a fermion, and has a sizable interaction with the SM particles so that it can be thermalized in the early Universe. The WIMP DM in SO(10) GUTs is thoroughly discussed in Ref. [2], and we briefly review the result in Sec. 3. We focus on SO(10) GUT models in which gauge coupling unification is achieved with a sufficiently high GUT scale to evade constraints coming from proton decay experiments. We also check whether these models yield the correct DM density and account for small neutrino masses.

Throughout this article, we consider the following symmetry-breaking chain,

$$
\mathrm{SO}(10) \longrightarrow G_{\text {int }} \longrightarrow \mathrm{SU}(3)_{C} \otimes \mathrm{SU}(2)_{L} \otimes \mathrm{U}(1)_{Y} \otimes \mathbb{Z}_{2}
$$

where the SO(10) GUT group is broken at the GUT scale $M_{\mathrm{GUT}}$ into the intermediate gauge group $G_{\text {int }}$ by a VEV of the GUT-scale Higgs multiplet $R_{1}$. This intermediate gauge group is subsequently broken to the SM gauge group at the intermediate scale $M_{\text {int }}$ by a VEV of the intermediate-scale Higgs multiplet $R_{2}$. The GUT and intermediate scales are determined using the condition of gauge coupling unification. We denote the DM multiplet by $R_{\mathrm{DM}}$. Among the components in $R_{\mathrm{DM}}$, only the DM field has a mass much lighter than the intermediate scale so that it can explain the observed DM density, while the other components are supposed to have masses of $\mathscr{O}\left(M_{\text {int }}\right)$. Such a mass spectrum is obtained by fine-tuning of the coefficients in the original Lagrangian terms, just like the ordinary doublet-triplet splitting in the Higgs sector. We only consider $\mathrm{SO}(10)$ multiplets whose dimensions are equal to or less than 210 in the following discussion.

\section{Singlet Fermion DM: NETDM}

First, we discuss the case of SM singlet fermion DM. This class of DM candidates cannot couple to the SM sector at renormalizable level. Therefore, if new physics appears at a very high scale ( $M_{\text {int }}$ in the present setup), the DM interactions with SM particles are so weak that this DM cannot be in thermal equilibrium in the early Universe. Nevertheless, such a DM particle can be produced via the NETDM mechanism [22] as we discuss below.

For a fermion to be stabilized by the $\mathbb{Z}_{2}$ symmetry in Eq. (1.2), it should have an even parity under the symmetry. It is found that a SM singlet fermion which has an even $\mathbb{Z}_{2}$ charge appears in a 45, 54, 126, or 210 of $S O(10)$. By requiring gauge coupling unification with a sufficiently high GUT scale, we have found two promising models which contain singlet fermion DM. ${ }^{1}$ We

\footnotetext{
${ }^{1}$ Here, we have assumed the DM multiplet to be charged under the intermediate gauge symmetry. In this case, the DM can be produced via the exchange of the intermediate-scale particles. We can also consider the case where the DM multiplet is also singlet under the intermediate gauge symmetry. This DM can still be produced via the GUT-scale interactions. Such a possibility is also discussed in Ref. [1].
} 


\begin{tabular}{l|cc}
\hline \hline & Model I & Model II \\
\hline$G_{\text {int }}$ & $\mathrm{SU}(4)_{C} \otimes \mathrm{SU}(2)_{L} \otimes \mathrm{SU}(2)_{R}$ & $\mathrm{SU}(4)_{C} \otimes \mathrm{SU}(2)_{L} \otimes \mathrm{SU}(2)_{R} \otimes D$ \\
$R_{\mathrm{DM}}$ & $(\mathbf{1}, \mathbf{1}, \mathbf{3})_{D}$ in $\mathbf{4 5}_{D}$ & $(\mathbf{1 5}, \mathbf{1}, \mathbf{1})_{W}$ in $\mathbf{4 5}_{W}$ \\
$R_{1}$ & $\mathbf{2 1 0}_{R}$ & $\mathbf{5 4}_{R}$ \\
$R_{2}$ & $(\mathbf{1 0}, \mathbf{1}, \mathbf{3})_{C} \oplus(\mathbf{1}, \mathbf{1}, \mathbf{3})_{R}$ & $(\mathbf{1 0}, \mathbf{1}, \mathbf{3})_{C} \oplus(\mathbf{1 0}, \mathbf{3}, \mathbf{1})_{C} \oplus(\mathbf{1 5}, \mathbf{1}, \mathbf{1})_{R}$ \\
$M_{\text {int }}$ & $1.2 \times 10^{8} \mathrm{GeV}$ & $4.6 \times 10^{13} \mathrm{GeV}$ \\
$M_{\mathrm{GUT}}$ & $4.4 \times 10^{15} \mathrm{GeV}$ & $7.4 \times 10^{15} \mathrm{GeV}$ \\
$g_{\mathrm{GUT}}$ & 0.53 & 0.57 \\
\hline \hline
\end{tabular}

Table 1: Singlet fermion DM models [1].

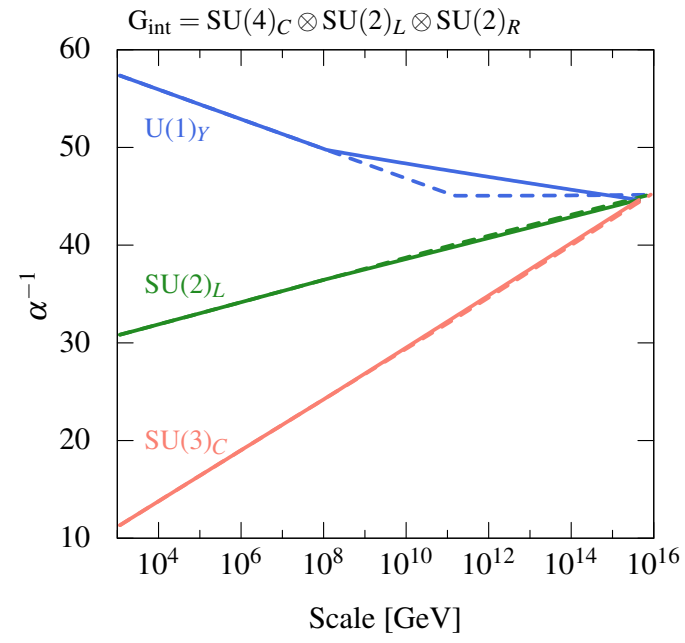

(a) Model I

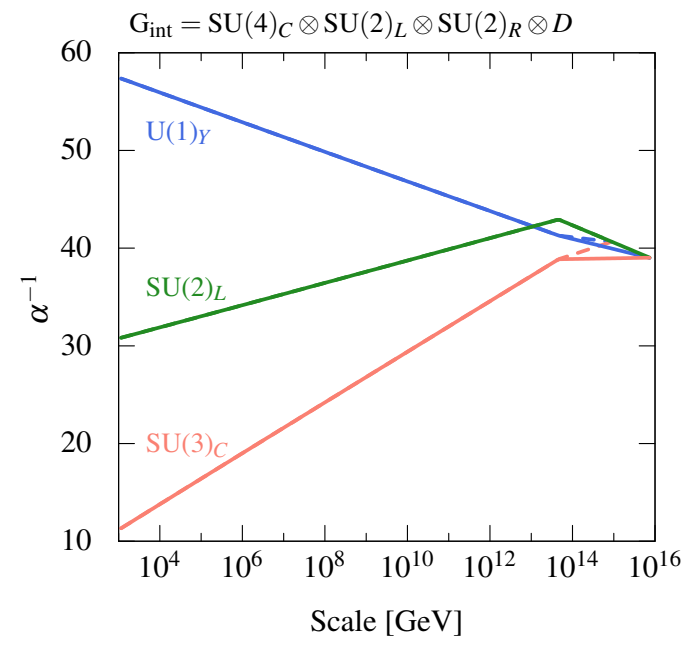

(b) Model II

Figure 1: Running of gauge couplings [1]. Solid (dashed) lines show the case with (without) DM and additional Higgs bosons. Blue, green, and red lines represent the running of the $\mathrm{U}(1)_{Y}, \mathrm{SU}(2)_{L}$ and $\mathrm{SU}(3)_{C}$ gauge couplings, respectively.

summarize the contents of these models in Table 1. One of the models has an intermediate gauge symmetry of $G_{\text {int }}=\mathrm{SU}(4)_{C} \otimes \mathrm{SU}(2)_{L} \otimes \mathrm{SU}(2)_{R}$, while the other has $G_{\text {int }}=\mathrm{SU}(4)_{C} \otimes \mathrm{SU}(2)_{L} \otimes$ $\mathrm{SU}(2)_{R} \otimes D$ with $D$ representing the left-right parity—an additional discrete symmetry with respect to the interchange of left- and right-handed fields. We refer to the former (latter) as Model I (II) in what follows. In Model I, the singlet fermion DM stays inside the $(\mathbf{1}, \mathbf{1}, \mathbf{3})_{D}$ component of a $\mathbf{4 5}_{D}$ of $\mathrm{SO}(10)$, while in Model II it is in the $(\mathbf{1 5}, \mathbf{1}, \mathbf{1})_{W}$ of a $\mathbf{4 5}_{W}$. Here, the subscripts $R, C, W$, and $D$ indicate a real scalar boson, a complex scalar boson, a Weyl fermion, and a Dirac fermion, respectively. The $(\mathbf{1 0}, \mathbf{1}, \mathbf{3})_{C}$ component appearing in $R_{2}$ originates from a $\mathbf{1 2 6}_{C}$, whose VEV yields the remnant $\mathbb{Z}_{2}$ symmetry. The other Higgs fields in $R_{2}$ are introduced to give masses of $\mathscr{O}\left(M_{\text {int }}\right)$ to all of the components in $R_{\mathrm{DM}}$ except the DM field. The GUT and intermediate scales as well as the unified gauge coupling $g_{\mathrm{GUT}}$ in Table 1 are evaluated by using the two-loop renormalization group equations (RGEs). We also show the running of the gauge couplings in these models in Fig. 1. 


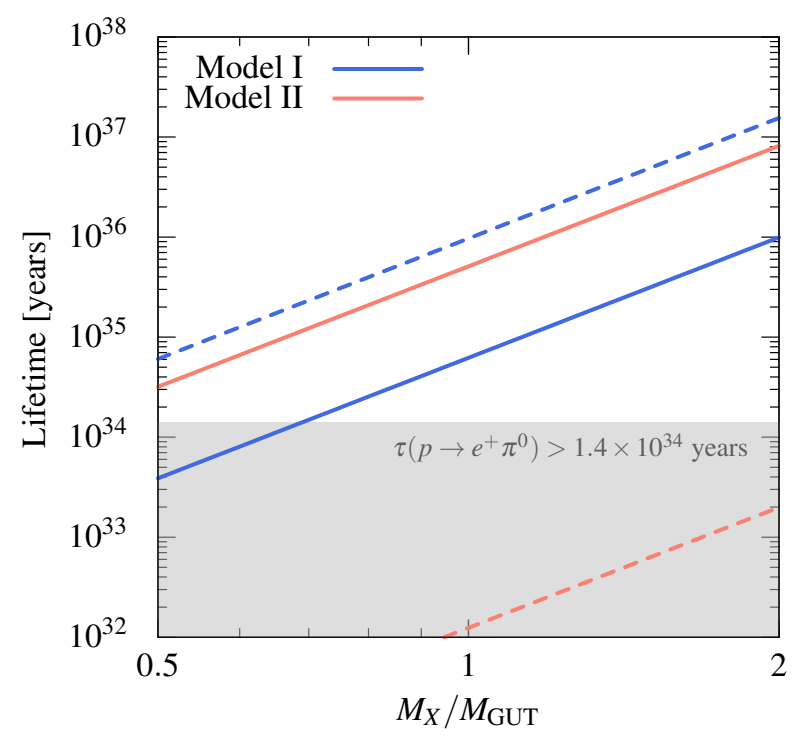

Figure 2: Proton lifetimes as functions of $M_{X} / M_{\mathrm{GUT}}$ [1]. Blue and red solid lines correspond to Model I and II, respectively. Blue and red dashed lines represent the cases for $G_{\text {int }}=\operatorname{SU}(4)_{C} \otimes \mathrm{SU}(2)_{L} \otimes \mathrm{SU}(2)_{R}$ and $G_{\text {int }}=\mathrm{SU}(4)_{C} \otimes \mathrm{SU}(2)_{L} \otimes \mathrm{SU}(2)_{R} \otimes D$, respectively, where the DM and extra Higgs multiplets are not included. Shaded region shows the current experimental bound, $\tau\left(p \rightarrow e^{+} \pi^{0}\right)>1.4 \times 10^{34}$ years [23].

Here, the solid and dashed lines show the cases with and without DM and additional intermediatescale Higgs multiplets, respectively. The $\mathrm{U}(1)_{Y}$ gauge coupling constant above the intermediate scale is defined by $\frac{1}{\alpha_{Y}} \equiv \frac{3}{5} \frac{1}{\alpha_{2 R}}+\frac{2}{5} \frac{1}{\alpha_{4}}$ where $\alpha_{2 R}\left(\alpha_{4}\right)$ is the $\mathrm{SU}(2)_{R}\left(\mathrm{SU}(4)_{C}\right)$ gauge coupling, while the $\mathrm{SU}(3)_{C}$ gauge coupling above $M_{\text {int }}$ is given by $\alpha_{3} \equiv \alpha_{4}$. As can be seen from the plots, the presence of the DM and extra intermediate-scale Higgs multiplets significantly affects the running of the gauge coupling constants.

As we have obtained the GUT scales in the models, we can now evaluate proton decay lifetimes for each model. In the non-SUSY GUTs, proton decay is induced by the exchange of the GUTscale gauge bosons. The dominant decay mode in this case is the $p \rightarrow e^{+} \pi^{0}$ process. A calculation of proton decay rates in $\mathrm{SO}(10)$ GUTs is presented in Appendix B in Ref. [2], and we follow the prescription given there. We show the resultant proton decay lifetimes in the models in Fig. 2. Here, the blue solid and red solid lines represent proton lifetimes in Model I and II, respectively, while the dashed lines correspond to the cases in which the DM and extra Higgs multiplets are not included. Proton lifetimes depend on the GUT-scale gauge boson mass $M_{X}$. Although $M_{X}$ is expected to be of the order of the GUT scale, its precise value cannot be determined from low energies, and this ignorance therefore causes uncertainty in the proton decay calculation. Considering this, we estimate the uncertainty by varying $M_{X}$ from $M_{\mathrm{GUT}} / 2$ to $2 M_{\mathrm{GUT}}$ in this plot. The gray shaded region in Fig. 2 shows the current experimental bound, $\tau\left(p \rightarrow e^{+} \pi^{0}\right)>1.4 \times 10^{34}$ years [23]. From this figure, we find that proton lifetimes for $M_{X} \simeq M_{\mathrm{GUT}}$ lie above the current constraint in both of the models. Notice that the presence of the DM and extra Higgs multiplets gives a significant impact on the prediction of proton lifetimes; indeed, in the absence of these particles, the $G_{\text {int }}=\mathrm{SU}(4)_{C} \otimes \mathrm{SU}(2)_{L} \otimes \mathrm{SU}(2)_{R} \otimes D$ case predicts too rapid proton decay rate to evade the 


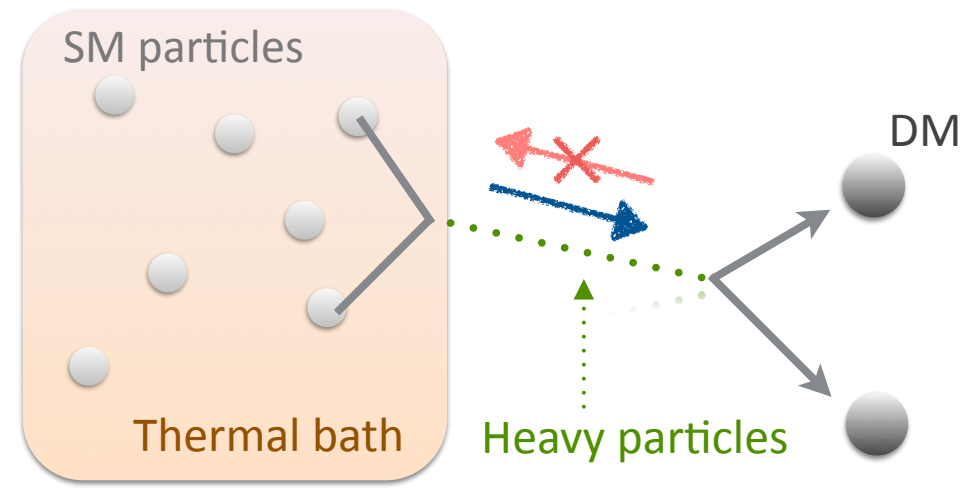

Figure 3: The NETDM mechanism [22] for DM production.

current experimental limit, but the addition of these particles makes Model II viable. Anyway, in both of these models, proton decay lifetimes are predicted to be rather short. Hence, these models may be probed in future proton decay experiments.

Next, we consider neutrino masses in these models. In both of the models, neutrino masses are given by the ordinary seesaw mechanism $[11,12,13,14,15,16]$. An important caveat here is that in the case of $\mathrm{SO}(10)$ GUTs Dirac Yukawa couplings for neutrinos are related to other Yukawa couplings. In particular, since the $\mathrm{SU}(4)_{C}$ symmetry is manifest above $M_{\text {int }}$ in our models and a VEV of the $(\mathbf{1}, \mathbf{2}, \mathbf{2})$ component, which is identified as the SM Higgs field in the minimal setup, does not break the $\mathrm{SU}(4)_{C}$ symmetry, the Dirac masses of neutrinos are equal to up-quark masses $m_{u_{i}}$ up to logarithmic corrections in the low-energy region. As a consequence, neutrino masses $m_{v_{i}}$ are given by

$$
m_{v_{i}} \simeq \frac{m_{u_{i}}^{2}}{M_{\text {int }}}
$$

where we have used the fact that Majorana masses for right-handed neutrinos are $\mathscr{O}\left(M_{\text {int }}\right)$. From the results given in Table 1, we then find that the Model II predicts neutrino masses compatible with the present data, while those in Model I are too large. This is because of a low intermediate scale in Model I, as can be seen from Fig. 1(a). In this sense, Model II is more favorable than Model I. A simple way to make Model I viable is to exploit the $(\mathbf{1 5 , 2 , 2})$ component of the $\mathbf{1 2 6}$ Higgs multiplet. This component contains an $\mathrm{SU}(2)_{L}$ doublet Higgs field, which can have a VEV of the order of the electroweak scale. Since this VEV breaks the $\mathrm{SU}(4)_{C}$ symmetry, one can choose its VEV and Yukawa couplings such that its contribution to Dirac mass terms of neutrinos cancels the above contribution. In this case, we can evade the relation (2.1), and desirable neutrino masses can be obtained even with a low intermediate scale. We also find that the presence of a $(\mathbf{1 5}, \mathbf{2}, \mathbf{2})$ field scarcely affects gauge coupling unification, as it changes the beta functions of all of the gauge coupling constants by similar amounts [24]. Therefore, the introduction of a $(\mathbf{1 5}, \mathbf{2}, \mathbf{2})$ resolves the neutrino mass problem in Model I without largely modifying the GUT and intermediate scales obtained above.

Now we consider the DM production mechanism in these models. As mentioned above, DM particles in these models do not come into thermal equilibrium in the early Universe as long as 


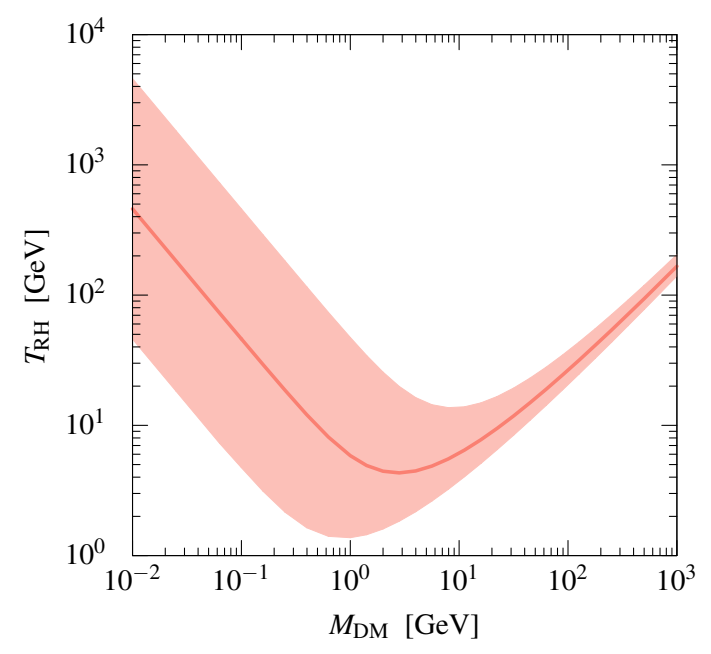

(a) Model I

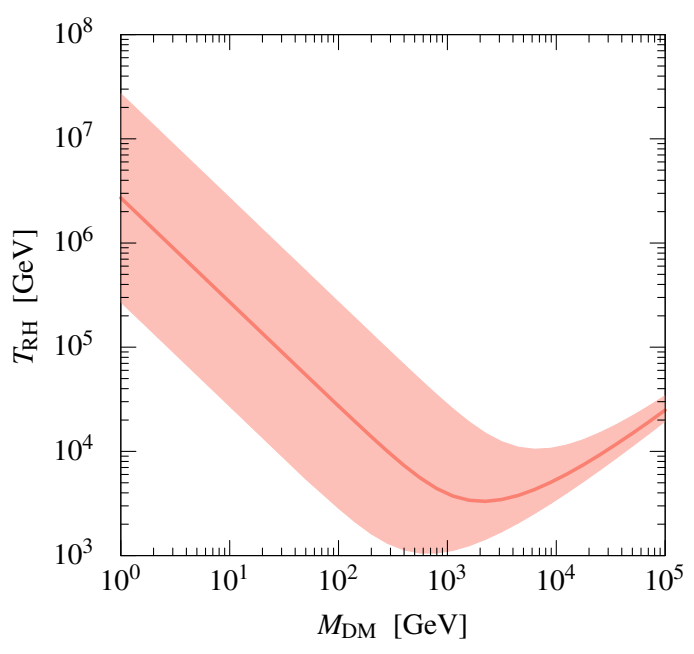

(b) Model II

Figure 4: Reheating temperature as a function of $M_{D M}$ [1]. Pink band shows the theoretical uncertainty.

the reheating temperature $T_{\mathrm{RH}}$ is lower than the intermediate scale. In this case, DM is produced non-thermally from the scattering of the SM particles in the thermal bath through the exchange of intermediate/GUT scale particles. The pair annihilations of DM particles during the DM production are negligible. This situation is illustrated in Fig. 3. The current DM abundance in this case is evaluated by using the following Boltzmann equation:

$$
\frac{d Y_{\mathrm{DM}}}{d x}=\sqrt{\frac{\pi g_{*}}{45}} M_{\mathrm{DM}} M_{\mathrm{Pl}} \frac{\langle\sigma v\rangle}{x^{2}} Y_{\mathrm{eq}}^{2},
$$

where $Y_{\mathrm{DM}} \equiv n_{\mathrm{DM}} / s, Y_{\mathrm{eq}} \equiv n_{\mathrm{eq}} / s, n_{\mathrm{DM}}$ is the number density of DM, $n_{\mathrm{eq}}$ is the equilibrium number density of each individual initial state SM particle, $s$ is the entropy of the Universe, $x \equiv M_{\mathrm{DM}} / T$, $T$ is the temperature of the Universe, $M_{\mathrm{DM}}$ is the DM mass, $M_{\mathrm{Pl}} \equiv 1 / \sqrt{G_{N}}=1.22 \times 10^{19} \mathrm{GeV}, g_{*}$ is the effective degrees of freedom in the thermal bath, and $\langle\sigma v\rangle$ denotes the thermally averaged total annihilation cross section of the initial SM particles into the DM pair. Notice that there is no self-annihilation contribution in the right-hand side in Eq. (2.2), as mentioned above. By solving this equation, we can obtain DM relic abundance as a function of $M_{\mathrm{DM}}$ and $T_{\mathrm{RH}}$.

In Fig. 4, we show the regions favored by the DM relic abundance in the $M_{\mathrm{DM}}-T_{\mathrm{RH}}$ plane for each model. Here, the pink bands indicate the uncertainty of our computation. We see that Model I predicts low value of $T_{\mathrm{RH}}$ except for the fine-tuning region $T_{\mathrm{RH}} \simeq M_{\mathrm{DM}}$. For such a low reheating temperature, baryogenesis is usually quite challenging. On the other hand, $T_{\mathrm{RH}}$ can be sufficiently high in the case of Model II. Thus, Model II is again favored compared to Model I.

\section{WIMP DM Candidates}

Next, we briefly discuss WIMP DM in the SO(10) GUT models. For systematic classification of such possibilities and detailed discussions on the models, see Ref. [2]. In the case of WIMP DM, we have both scalar and fermion candidates. For a scalar particle to be stable, it should 
have odd matter parity, which can originate from either a 16 or $\mathbf{1 4 4}$ of $\mathrm{SO}(10)$. By requiring a sufficiently high unification scale to ensure a proton decay lifetime compatible with the current experimental limits, we find that only the SM singlet DM or the $\mathrm{SU}(2)_{L}$ doublet with hypercharge $Y= \pm 1 / 2$ scalar DM can be realized in the low-energy regions, with possible intermediate scales being $\mathrm{SU}(4)_{C} \otimes \mathrm{SU}(2)_{L} \otimes \mathrm{SU}(2)_{R}, \mathrm{SU}(3)_{C} \otimes \mathrm{SU}(2)_{L} \otimes \mathrm{SU}(2)_{R} \otimes \mathrm{U}(1)_{B-L}$, or $\mathrm{SU}(3)_{C} \otimes \mathrm{SU}(2)_{L} \otimes$ $\mathrm{SU}(2)_{R} \otimes \mathrm{U}(1)_{B-L} \otimes D$. The former case, in which a singlet scalar is added to the SM as a DM candidate, is one of the simplest extensions of the SM and has widely been discussed so far [25, 26, 27]. The latter DM candidate is often called the inert Higgs doublet DM [28, 29, 30, 31]. As for fermionic DM candidates, we obtain an $\mathrm{SU}(2)_{L}$ triplet $Y=0 \mathrm{DM}$ candidate from a 45 of $\mathrm{SO}(10)$, though it requires additional Higgs multiplets around the intermediate scale to realize good gauge coupling unification. We can also find $\mathrm{SU}(2)_{L}$ doublet DM candidates. Such a DM candidate in general requires extra Majorana fermions beyond the DM multiplet; otherwise the DM candidate becomes a Dirac fermion and such a possibility has already been excluded by direct detection experiments since Dirac fermion DM with non-zero hypercharge has a large DM-nucleon scattering cross section. To evade the constraint, the $\mathrm{SU}(2)_{L}$ doublet DM has to mix with the extra Majorana fermions to be split into two pseudo-Dirac fermions. Moreover, to also suppress the inelastic scattering of the DM with nucleons, the mass difference between these pseudo-Dirac fermions should be $\gtrsim 100 \mathrm{keV}$ [32]. This condition gives an upper bound on the masses of the extra Majorana fermions [33], and if we assume these masses to be $\mathscr{O}\left(M_{\text {int }}\right)$, this bound leads to an upper bound on $M_{\text {int }}$. Taking into account this constraint, as well as that from proton decay bounds, we found several models for the $\mathrm{SU}(2)_{L}$ doublet DM, which turn out to have either $G_{\text {int }}=$ $\mathrm{SU}(4)_{C} \otimes \mathrm{SU}(2)_{L} \otimes \mathrm{SU}(2)_{R}$ or $\mathrm{SU}(4)_{C} \otimes \mathrm{SU}(2)_{L} \otimes \mathrm{U}(1)_{R}$. These non-SUSY SO(10) WIMP DM models can be tested in future DM direct searches and proton decay experiments.

\section{Conclusion and Discussions}

In this work, we have discussed DM candidates in non-SUSY SO(10) GUT models with an intermediate gauge symmetry. In these models, the stability of DM is achieved thanks to a remnant $\mathbb{Z}_{2}$ symmetry which is a subgroup of SO(10). The DM can be either NETDM or WIMP DM. For both cases, the requirement of gauge coupling unification with a sufficiently high GUT scale to evade the proton decay limit plays a significant role in selecting viable candidates. In the case of NETDM, we found two promising models which satisfy the above condition. The DM particles in these models are non-thermally produced from the scattering of the SM particles in the thermal bath via the exchange of intermediate-scale particles; we have computed the favored reheating temperature which yields the correct DM density. As for the WIMP DM case, we have found models that include a SM singlet, an inert Higgs doublet, an $\mathrm{SU}(2)_{L}$ triplet, or an $\mathrm{SU}(2)_{L}$ doublet DM candidate. In any cases, future DM direct searches and proton decay experiments can probe these DM models.

Finally, we would like to comment on some previous studies. In Refs. [18, 19], a SM singlet scalar and $\mathrm{SU}(2)_{L}$ doublet scalar DM originating from a 16 of $\mathrm{SO}(10)$ are considered, where $\mathrm{SO}(10)$ is assumed to be broken to $\mathrm{SU}(5) \otimes \mathrm{U}(1)_{X}$ and this $\mathrm{U}(1)_{X}$ is broken to the $\mathbb{Z}_{2}$ symmetry. Gauge coupling unification and the proton decay constraints are not discussed in detail. In Ref. [20], on the other hand, both scalar and fermion DM candidates are discussed. Intermediate gauge symmetries 
are not discussed there, and for this reason, an $\mathrm{SU}(2)_{L}$ triplet with $Y=0$ is found to be the only promising candidate for DM which may make the unification scale high enough, though gauge coupling unification is challenging if one focuses on minimal field contents.

\section{Acknowledgments}

The work of the author was supported by Research Fellowships of the Japan Society for the Promotion of Science for Young Scientists.

\section{References}

[1] Y. Mambrini, N. Nagata, K. A. Olive, J. Quevillon, and J. Zheng, Dark matter and gauge coupling unification in nonsupersymmetric SO(10) grand unified models, Phys. Rev. D91 (2015), no. 9 095010, [arXiv:1502.06929].

[2] N. Nagata, K. A. Olive, and J. Zheng, Weakly-Interacting Massive Particles in Non-supersymmetric SO(10) Grand Unified Models, arXiv:1509.00809.

[3] T. W. B. Kibble, G. Lazarides, and Q. Shafi, Strings in SO(10), Phys. Lett. B113 (1982) 237.

[4] L. M. Krauss and F. Wilczek, Discrete Gauge Symmetry in Continuum Theories, Phys. Rev. Lett. 62 (1989) 1221.

[5] L. E. Ibanez and G. G. Ross, Discrete gauge symmetries and the origin of baryon and lepton number conservation in supersymmetric versions of the standard model, Nucl. Phys. B368 (1992) 3-37.

[6] L. E. Ibanez and G. G. Ross, Discrete gauge symmetry anomalies, Phys. Lett. B260 (1991) 291-295.

[7] S. P. Martin, Some simple criteria for gauged R-parity, Phys. Rev. D46 (1992) 2769-2772, [hep-ph/9207218].

[8] H. Georgi, The State of the Art-Gauge Theories, AIP Conf. Proc. 23 (1975) 575-582.

[9] H. Fritzsch and P. Minkowski, Unified Interactions of Leptons and Hadrons, Annals Phys. 93 (1975) 193-266.

[10] M. Fukugita and T. Yanagida, Physics of neutrinos, in Physics and Astrophysics of Neutrinos (M. Fukugita and A. Suzuki, eds.). Springer Japan, 1994.

[11] P. Minkowski, $\mu \rightarrow$ e $\gamma$ at a Rate of One Out of $10^{9}$ Muon Decays?, Phys. Lett. $\mathbf{B 6 7}$ (1977) 421-428.

[12] T. Yanagida, HORIZONTAL SYMMETRY AND MASSES OF NEUTRINOS, Conf. Proc. C7902131 (1979) 95-99. [Conf. Proc.C7902131,95(1979)].

[13] M. Gell-Mann, P. Ramond, and R. Slansky, Complex Spinors and Unified Theories, Conf. Proc. C790927 (1979) 315-321, [arXiv:1306.4669].

[14] S. L. Glashow, The Future of Elementary Particle Physics, NATO Sci. Ser. B 61 (1980) 687.

[15] R. N. Mohapatra and G. Senjanovic, Neutrino Mass and Spontaneous Parity Violation, Phys. Rev. Lett. 44 (1980) 912.

[16] J. Schechter and J. W. F. Valle, Neutrino Masses in SU(2) $\times$ U(1) Theories, Phys. Rev. D22 (1980) 2227.

[17] M. De Montigny and M. Masip, Discrete gauge symmetries in supersymmetric grand unified models, Phys. Rev. D49 (1994) 3734-3740, [hep-ph/ 9309312 ]. 
[18] M. Kadastik, K. Kannike, and M. Raidal, Matter parity as the origin of scalar Dark Matter, Phys. Rev. D81 (2010) 015002, [arXiv: 0903.2475$].$

[19] M. Kadastik, K. Kannike, and M. Raidal, Dark Matter as the signal of Grand Unification, Phys. Rev. D80 (2009) 085020, [arXiv: 0907.1894 ]. [Erratum: Phys. Rev.D81,029903(2010)].

[20] M. Frigerio and T. Hambye, Dark matter stability and unification without supersymmetry, Phys. Rev. D81 (2010) 075002, [arXiv:0912.1545].

[21] T. Hambye, On the stability of particle dark matter, PoS IDM2010 (2011) 098, [arXiv:1012.4587].

[22] Y. Mambrini, K. A. Olive, J. Quevillon, and B. Zaldivar, Gauge Coupling Unification and Nonequilibrium Thermal Dark Matter, Phys. Rev. Lett. 110 (2013), no. 24 241306, [arXiv:1302.4438].

[23] K. S. Babu et al., Working Group Report: Baryon Number Violation, in Community Summer Study 2013: Snowmass on the Mississippi (CSS2013) Minneapolis, MN, USA, July 29-August 6, 2013, 2013. arXiv:1311.5285.

[24] B. Bajc, A. Melfo, G. Senjanovic, and F. Vissani, Yukawa sector in non-supersymmetric renormalizable SO(10), Phys. Rev. D73 (2006) 055001, [hep-ph / 0510139 ].

[25] V. Silveira and A. Zee, SCALAR PHANTOMS, Phys. Lett. B161 (1985) 136.

[26] J. McDonald, Gauge singlet scalars as cold dark matter, Phys. Rev. D50 (1994) 3637-3649, [hep-ph/0702143].

[27] C. P. Burgess, M. Pospelov, and T. ter Veldhuis, The Minimal model of nonbaryonic dark matter: A Singlet scalar, Nucl. Phys. B619 (2001) 709-728, [hep-ph/0 011335$].$

[28] N. G. Deshpande and E. Ma, Pattern of Symmetry Breaking with Two Higgs Doublets, Phys. Rev. D18 (1978) 2574.

[29] E. Ma, Verifiable radiative seesaw mechanism of neutrino mass and dark matter, Phys. Rev. D73 (2006) 077301, [hep-ph/0601225].

[30] R. Barbieri, L. J. Hall, and V. S. Rychkov, Improved naturalness with a heavy Higgs: An Alternative road to LHC physics, Phys. Rev. D74 (2006) 015007, [hep-ph/ 0603188 ].

[31] L. Lopez Honorez, E. Nezri, J. F. Oliver, and M. H. G. Tytgat, The Inert Doublet Model: An Archetype for Dark Matter, JCAP 0702 (2007) 028, [hep-ph / 0612275$].$

[32] N. Nagata and S. Shirai, Higgsino Dark Matter in High-Scale Supersymmetry, JHEP 01 (2015) 029, [arXiv:1410.4549].

[33] N. Nagata and S. Shirai, Electroweakly-Interacting Dirac Dark Matter, Phys. Rev. D91 (2015), no. 5 055035, [arXiv:1411.0752]. 